\title{
Complicações relacionadas à punção venosa periférica e à hipodermóclise em pacientes oncológicos sob cuidados paliativos
}

Complications related to peripheral venipuncture and hypodermoclysis in cancer patients under palliative care Complicaciones relacionadas con la venopunción periférica y la hipodermoclisis en pacientes oncológicos en cuidados paliativos

\section{Ana Julia de Oliveira Lago', Ana Carolina de Souza ${ }^{\mathrm{II}}$, Fabiana Bolela ${ }^{\mathrm{II}}$}

\begin{abstract}
Resumo: Objetivo: identificar complicações relacionadas à punção venosa periférica e à hipodermóclise em pacientes oncológicos hospitalizados sob cuidados paliativos. Método: estudo descritivo e longitudinal. Foi utilizado questionário para caracterização sociodemográfica e clínica dos participantes, roteiro para a avaliação e acompanhamento da punção. Os dados foram analisados por meio da estatística descritiva. Resultados: participaram do estudo 70 pacientes, sendo $54,3 \%(\mathrm{n}=38)$ do sexo feminino e com idade média de 68,8 anos $(\mathrm{DP}=$ 15,0), cujas principais doenças oncológicas de base foram câncer de cabeça e pescoço e de pulmão. Ademais, $90 \%$ das punções avaliadas foram endovenosas e as complicações mais frequentes foram dor local, extravasamento e cateter dobrado ou tracionado. Conclusão: as complicações observadas no período do estudo foram relacionadas apenas às punções venosas. Houve predominância da punção venosa periférica em detrimento da via subcutânea, uma alternativa viável e recomendada para os pacientes oncológicos sob cuidados paliativos.

Descritores: Cuidados Paliativos; Hipodermóclise; Infusões Subcutâneas; Cateterismo Periférico; Oncologia
\end{abstract}

Abstract: Objective: to identify complications related to peripheral venipuncture and hypodermoclysis in cancer patients hospitalized under palliative care. Method: this is a descriptive and longitudinal study. A questionnaire was used for the sociodemographic and clinical characterization of participants, a script for puncture assessment and monitoring. The data were ran through descriptive statistics. Results: seventy patients participated in the study, $54.3 \%$ ( $\mathrm{n}=38$ ) of whom were female and with a mean age of 68.8 years $(\mathrm{SD}=15.0$ ), whose main underlying oncological diseases were head cancer and neck and lung. Furthermore, $90 \%$ of assessed punctures were intravenous and the most frequent complications were localized pain, extravasation and a bent or pulled catheter. Conclusion: the complications observed during the study period were related only to venipunctures. There was a

\footnotetext{
I Enfermeira, Escola de Enfermagem de Ribeirão Preto da Universidade de São Paulo (USP), Ribeirão Preto, SP, Brasil. E-mail: ana.julia.lago@usp.br, Orcid: https://orcid.org/0000-0003-0933-1917

II Enfermeira, Pós-graduada em Cuidados Paliativos, Mestranda em Enfermagem da Escola de Enfermagem de Ribeirão Preto da Universidade de São Paulo (USP), Ribeirão Preto, SP, Brasil. E-mail: ana3.souza@usp.br, Orcid: https://orcid.org/0000-0003-4126-4224

III Enfermeira, Doutora em Ciências, Docente na Escola de Enfermagem de Ribeirão Preto da Universidade de São Paulo (USP), Ribeirão Preto, SP, Brasil. E-mail: fbolela@usp.br, Orcid: https://orcid.org/0000-0003-1199-6205
} 
predominance of peripheral venipuncture due to the subcutaneous route, a viable and recommended alternative for cancer patients under palliative care.

Descriptors: Palliative Care; Hypodermoclysis; Infusions, Subcutaneous; Catheterization, Peripheral; Medical Oncology

Resumen: Objetivo: identificar las complicaciones relacionadas con la venopunción periférica y la hipodermoclisis en pacientes con cáncer hospitalizados bajo cuidados paliativos. Método: estudio descriptivo y longitudinal. Se utilizó un cuestionario para la caracterización sociodemográfica y clínica de los participantes, un guión para la valoración y seguimiento de la punción. Los datos fueron analizados utilizando estadística descriptiva. Resultados: en el estudio participaron 70 pacientes, de los cuales el 54,3\% (n=38) eran mujeres y con una edad media de 68,8 años ( $\mathrm{DE}=15,0)$, cuyas principales patologías oncológicas de base fueron cabeza y cuello y pulmón. Además, el $90 \%$ de las punciones evaluadas fueron intravenosas y las complicaciones más frecuentes fueron dolor local, extravasación y catéter doblado o tirado. Conclusión: las complicaciones observadas durante el período de estudio se relacionaron únicamente con las punciones venosas. Predominó la punción venosa periférica en detrimento de la vía subcutánea, alternativa viable y recomendada para pacientes oncológicos en cuidados paliativos.

Descriptores: Cuidados Paliativos; Hipodermoclisis; Infusiones Subcutáneas; Cateterismo Periférico; Oncología Médica

\section{Introdução}

Os cuidados paliativos (CP) são oferecidos, principalmente, a pessoas que estão no final da vida, sem restrição de idade, que, devido a doenças graves, enfrentam sofrimento intenso relativo à saúde. O objetivo dos CP é oferecer cuidados holísticos ativos e melhorar a qualidade de vida dos pacientes, de suas famílias e de seus cuidadores. ${ }^{1}$ Diante do crescimento da população idosa e do aumento das doenças crônico-degenerativas, como o câncer, as discussões sobre métodos alternativos para administração de medicamentos e soluções de reidratação têm crescido, como é o caso do uso da via subcutânea, quando as vias endovenosa e oral se apresentam limitadas, e de procedimentos invasivos, tais como dissecação venosa e inserção de cateteres, quando são contraindicados. ${ }^{2}$

A punção venosa periférica (PVP) é uma terapia intravenosa realizada rotineiramente no cuidado hospitalar por meio de aparatos tecnológicos, entre eles os cateteres intravenosos periféricos. $^{3}$ É uma técnica indicada para a administração de fármacos, fluidos, componentes sanguíneos e nutricionais, utilizada em pacientes que precisam de terapia intravenosa para tratar patologias em diferentes circunstâncias. O cateter mais utilizado é o cateter venoso periférico (CVP), que permite a aplicação de medicações ou soluções, nutrição parenteral, hemoderivados e 
coleta de sangue..$^{3-4}$ Apesar de ser amplamente utilizado, o CVP pode acarretar no desenvolvimento de complicações locais e sistêmicas, havendo evidências acerca da elevada incidência de reações locais após sua inserção, destacando-se, entre essas, a flebite e a infiltração. ${ }^{4}$

Já a hipodermóclise consiste na administração de medicamentos e fluidos no espaço subcutâneo, de forma contínua ou intermitente. ${ }^{2}$ É descrita como um procedimento simples, seguro e sem complicações graves, porém ainda é uma técnica pouco conhecida e utilizada no cenário hospitalar, existindo questionamentos quanto à sua utilização, quais medicamentos e fluidos podem ser seguramente administrados, em que volume e velocidade de infusão, entre outros aspectos. ${ }^{5-6}$

As complicações relacionadas ao uso da via subcutânea são raras quando é adotado o uso correto do procedimento de punção, da diluição e da infusão de medicamentos. ${ }^{7}$ Segundo revisão integrativa sobre os efeitos adversos encontrados em hipodermóclises realizadas em adultos, as reações mais relatadas nos estudos foram dor e edema no local da punção, celulite e absorção insuficiente da solução com acúmulo de líquido no local. Os riscos citados apresentam-se como sendo reversíveis e de pouca importância clínica, que podem ser tratados com massagem local, diminuição na taxa de infusão e mudança do sítio de punção. No Brasil, ainda existem poucos estudos e protocolos sobre o uso da hipodermóclise, sendo necessário o desenvolvimento de pesquisas que abordem o método proposto como uma alternativa para os pacientes, incluindo as questões relacionadas aos efeitos adversos, segurança do paciente, efetividade da técnica e sua repercussão na qualidade de vida dos pacientes..$^{6,8-9}$

Diante do exposto, tem-se como objetivo identificar complicações relacionadas à PVP e à hipodermóclise em pacientes oncológicos hospitalizados sob CP.

\section{Método}

Trata-se de estudo descritivo e longitudinal, realizado em duas enfermarias de clínica médica de um hospital de nível secundário no interior do estado de São Paulo. A população da 
presente pesquisa correspondeu aos pacientes oncológicos internados sob $\mathrm{CP}$, acompanhados por equipes inter-consultoras de CP. Os critérios de inclusão foram ser pacientes com idade superior a 18 anos, com diagnóstico de doença oncológica, internados sob CP (com o registro do CID 10 - Z51.5 em prontuário) e com a necessidade de punção para terapia medicamentosa parenteral. O critério de exclusão foi ausência de familiar ou cuidador para responder pelo paciente nos casos em que ele apresente impossibilidade de responder aos instrumentos de coleta.

Os potenciais participantes foram convidados a participar da pesquisa por meio de abordagem direta realizada pelo pesquisador. Após concordarem, foi realizada a leitura do Termo de Consentimento Livre e Esclarecido (TCLE), impresso em duas vias. Uma via era entregue ao paciente, e a outra, arquivada pela pesquisadora após o consentimento e respectiva assinatura. Em situações em que o paciente não estivesse apto a consentir sua participação no estudo (sonolência, sedação), o consentimento e a assinatura do TCLE eram obtidos junto ao seu responsável legal.

A coleta de dados foi realizada de setembro de 2019 a fevereiro de 2020, por meio de entrevista com o paciente e/ou seu responsável legal, consulta ao prontuário, avaliação direta e acompanhamento diário da punção (venosa ou subcutânea), desde sua obtenção até o momento em que não houvesse mais indicação da punção ou alta/óbito do paciente. Foram utilizados instrumentos para a obtenção dos dados sociodemográficos e clínicos do paciente e roteiro para a avaliação e acompanhamento da punção.

Para a obtenção dos aspectos sociodemográficos, foram coletados os seguintes dados: idade, sexo, estado civil, anos completos de estudo formal, principal cuidador, grau de parentesco com o paciente e anos completos de estudo formal do cuidador. Para a caracterização clínica, foram obtidas as seguintes informações mediante consulta aos prontuários: data da internação, motivo da internação, doença oncológica de base, data da indicação de CP exclusivos, tratamento modificador da doença a que o paciente foi previamente 
submetido, datas do início e término do tratamento modificador, finalidade da PVP ou hipodermóclise e medicamentos prescritos para administração parenteral.

Avaliação e observação diária e direta da punção foi realizada por estudantes de graduação, enfermeiros ou professores devidamente capacitados, por meio de um roteiro construído pelos autores do estudo, contendo tipo de punção (venosa ou hipodermóclise), número de tentativas de punção, data da punção, local da punção, tipo de cateter e calibre, presença ou não de fita para estabilização do cateter, tipo de cobertura, identificação da punção (considerada presente ao conter data e horário de realização, nome do profissional que realizou e calibre do cateter utilizado), sinais de infecção no local de inserção do cateter (dor, calor local, hiperemia, edema, existência de secreção na inserção do cateter), necessidade de troca da punção e motivo, inviabilidade da manutenção da punção.

Os dados foram estruturados em planilhas do programa Microsoft Excel, passando por dupla digitação e uma etapa de validação para minimizar erros de transcrição. Para a caracterização sociodemográfica e clínica, utilizaram-se estatísticas descritivas, com o intuito de sumarizar as informações de interesse. Descreveram-se as variáveis qualitativas em termos de frequência e percentual, e as variáveis quantitativas, utilizando medidas de tendência central (média) e dispersão (desvio padrão).

Os dados foram processados e analisados por meio do programa estatístico IBM Statistical Package for the Social Sciences (SPSS), versão 24.0, para Windows. O projeto foi elaborado de acordo com os preceitos da Resolução CNS 466/12 e foi aprovado pelo Comitê de Ética em Pesquisa da Escola de Enfermagem de Ribeirão Preto da Universidade de São Paulo, em 20 de setembro de 2018, sob o Parecer n⿳ 2.912.046 e CAAE 91320318.1.3002.5440. ${ }^{10}$

\section{Resultados}

A amostra foi constituída por 70 participantes, cuja caracterização sociodemográfica está apresentada na Tabela 1. Ao todo, foram convidados a participar do estudo 86 pacientes que se 
\begin{tabular}{ll|l|l} 
Complicações relacionadas à punção venosa periférica e à hipodermóclise... & 6
\end{tabular}

enquadravam nos critérios de inclusão; entretanto, houve 16 recusas, a maior parte delas relacionada ao fato de o paciente estar se sentindo indisposto no momento ou por não desejar fazer parte da pesquisa.

Tabela 1 - Caracterização sociodemográfica dos pacientes oncológicos sob cuidados paliativos. Ribeirão Preto - SP, 2019. (n=70)

\begin{tabular}{lccc}
\hline \multicolumn{1}{c}{ Variáveis } & Média (DP) & $\mathbf{n}$ & $\%$ \\
\hline Idade & $68,8(15,0)$ & & \\
Sexo & & & \\
$\quad$ Feminino & & 38 & 54,3 \\
$\quad$ Masculino & 32 & 45,7 \\
Estado civil & & \\
$\quad$ Casado/União consensual & & 38 & 54,3 \\
$\quad$ Viúvo & & 13 & 18,6 \\
Separado/Divorciado & 10 & 14,3 \\
Solteiro & & 9 & 12,9 \\
Anos de estudo formal (n=64) & $5,8(4,3)$ & & \\
\hline
\end{tabular}

A caracterização clínica dos participantes está apresentada na Tabela 2. As principais doenças oncológicas de base foram cabeça e pescoço (11,4\%; n=8), pulmão $(11,4 \% ; n=8)$, mama $(10,0 \%$; $n=7)$ e colorretal $(10,0 \% ; n=7)$. As metástases mais prevalentes foram pulmonar $(25,7 \% ; n=18)$, óssea $(24,3 \%$; n=17) e hepática (21,4\%; n=15). Dentre as principais comorbidades, estão Hipertensão Arterial Sistêmica (HAS) (47,1\%; n=33), Diabetes Mellitus (27,1\%; n=19) e Dislipidemia (14,3\%; n=10).

Tabela 2 - Caracterização clínica dos pacientes oncológicos sob cuidados paliativos. Ribeirão Preto - SP, 2019. $(\mathrm{n}=70)$

\begin{tabular}{lcc}
\hline \multicolumn{1}{c}{ Variáveis } & $\mathbf{n}$ & $\mathbf{\%}$ \\
\hline Doença oncológica de base & & \\
Cabeça e pescoço & 8 & 11,4 \\
Pulmão & 8 & 11,4 \\
Mama & 7 & 10,0 \\
Colorretal & 7 & 10,0 \\
Próstata & 6 & 8,6 \\
Esôfago & 5 & 7,1 \\
Outras & 27 & 39,9 \\
Metástases & & \\
Pulmonar & 18 & 25,7 \\
Óssea & 17 & 24,3 \\
Hepática & 15 & 21,4 \\
Linfonodos & 8 & 11,4 \\
\hline
\end{tabular}




\begin{tabular}{lcc}
\hline SNC & 6 & 8,6 \\
Outras & 30 & 42,6 \\
Comorbidades & & \\
HAS & 33 & 47,1 \\
DM & 19 & 27,1 \\
Dislipidemia (DLP) & 10 & 14,3 \\
Hipotireoidismo & 9 & 12,9 \\
DPOC & 6 & 8,6 \\
Outras & 17 & 24,3
\end{tabular}

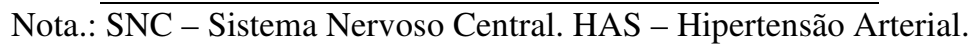

DM - Diabetes Mellitus. DPOC - Doença Pulmonar Obstrutiva Crônica

Durante o período do estudo, foram avaliadas 200 punções, dentre as quais $180(90,0 \%)$ eram punções venosas periféricas e 20 (10,0\%), punções subcutâneas (hipodermóclises). As características das punções avaliadas estão apresentadas na Tabela 3.

Tabela 3 - Características das punções em pacientes oncológicos sob cuidados paliativos. Ribeirão Preto - SP, 2019. (n=70)

\begin{tabular}{|c|c|c|}
\hline Variáveis & $\mathbf{n}$ & $\%$ \\
\hline \multicolumn{3}{|l|}{ Tipo de punção } \\
\hline Punção venosa periférica & 180 & 90,0 \\
\hline Punção Subcutânea & 20 & 10,0 \\
\hline \multicolumn{3}{|l|}{ Número de tentativas de punção venosa } \\
\hline 1 & 69 & 55,7 \\
\hline 2 & 34 & 27,4 \\
\hline 3 & 12 & 9,7 \\
\hline 4 & 5 & 4,0 \\
\hline 5 & 4 & 3,2 \\
\hline \multicolumn{3}{|l|}{ Local da punção endovenosa } \\
\hline Metacarpiana dorsal & 38 & 20,8 \\
\hline Cefálica & 33 & 18,0 \\
\hline Basílica & 24 & 13,1 \\
\hline Mediana cubital & 31 & 16,9 \\
\hline Braquial & 31 & 16,9 \\
\hline Plexo dorsal & 15 & 8,2 \\
\hline \multicolumn{3}{|l|}{ Região da punção subcutânea } \\
\hline Anterolateral da coxa & 12 & 60,0 \\
\hline Abdominal & 5 & 25,0 \\
\hline Deltoidea & 3 & 15,0 \\
\hline \multicolumn{3}{|l|}{ Tipo de cateter } \\
\hline Não agulhado (teflon) tipo Abocath ${ }^{\circledR}$ & 192 & 93,7 \\
\hline
\end{tabular}


Complicações relacionadas à punção venosa periférica e à hipodermóclise... $\mid 8$

\begin{tabular}{lcc}
\hline Não agulhado (teflon) tipo Intima & 13 & 6,3 \\
Calibre do cateter & 139 & 69,5 \\
22 & 46 & 23,0 \\
20 & 15 & 7,5 \\
24 & & \\
Fita de estabilização do cateter (se punção venosa) & 128 & 73,1 \\
Sim & 47 & 26,9 \\
Não & & \\
Tipo de cobertura & 189 & 91,3 \\
Filme transparente estéril & 17 & 8,2 \\
Associação de coberturas & 1 & 0,5 \\
Esparadrapo & & \\
Identificação da punção & 171 & 84,7 \\
Incompleta & 26 & 12,9 \\
Sim & 5 & 2,5 \\
Não & & \\
Finalidade da Punção & 33 & 11,8 \\
Antibioticoterapia & 32 & 11,4 \\
Analgesia & 12 & 4,3 \\
Hidratação & 11 & 3,9 \\
Controle de dispneia & 8 & 2,9 \\
Controle de náuseas e vômitos & 3 & 1,1 \\
Controle dos sintomas neurológicos & 1 & 0,4 \\
Melhora da constipação & & \\
\hline
\end{tabular}

$\mathrm{Na}$ Tabela 4, estão apresentados os principais medicamentos utilizados pelos participantes do estudo. A classe de medicamentos mais indicada foi a de analgésicos, com a dipirona como medicamento mais prescrito $(12,1 \% ; n=34)$, seguido de soroterapia para hidratação $(10,7 \%$; $n=30)$, corticoide dexametasona $(7,9 \% ; n=22)$ e o antiemético ondansetrona $(6,4 \% ; n=18)$.

Tabela 4 - Medicamentos mais utilizados pelos pacientes oncológicos sob cuidados paliativos. Ribeirão Preto - SP, 2019. (n=70)

\begin{tabular}{lcc}
\hline \multicolumn{1}{c}{ Medicamentos } & $\mathbf{n}$ & $\mathbf{\%}$ \\
\hline Dipirona & 34 & 12,1 \\
Soroterapia para hidratação & 30 & 10,7 \\
Dexametasona & 22 & 7,9 \\
Ondansetrona & 18 & 6,4 \\
Ceftriaxona & 13 & 4,6 \\
Morfina solução em bomba de infusão contínua & 11 & 3,9 \\
Bromoprida & 8 & 2,9
\end{tabular}


9 | Lago AJO, Souza ACS, Souza FB

\begin{tabular}{lcc}
\hline Morfina em bolus & 8 & 2,9 \\
Meropenem & 8 & 2,9 \\
Outros & 61 & 22,1 \\
\hline
\end{tabular}

Os motivos de internação estão apresentados na Tabela 5, sendo os principais motivos relacionados a alterações respiratórias $(6,8 \% ; \mathrm{n}=19)$, como dispneia, desconforto respiratório e dependência de oxigênio, e dor $(6,8 \% ; n=19)$, seguidos de sintomas relacionados à adinamia $(3,6 \% ; n=10)$.

Tabela 5 - Principais motivos das internações entre os pacientes oncológicos sob cuidados paliativos. Ribeirão Preto - SP, 2019. $(n=70)$

\begin{tabular}{lcc}
\hline \multicolumn{1}{c}{ Motivo da internação } & N & $\mathbf{\%}$ \\
\hline Dispneia/Desconforto respiratório & 19 & 6,8 \\
Dor & 19 & 6,8 \\
Adinamia/Rebaixamento ou alteração do nível de & & \\
consciência/Arresponsividade/Prostração/Sonolência & 10 & 3,6 \\
Queda/Piora do estado geral & 9 & 3,2 \\
Náuseas/Vômito & 9 & 3,2 \\
Pneumonia & 8 & 2,9 \\
Término de antibioticoterapia & 6 & 2,1 \\
Astenia/Perda de força & 6 & 2,1 \\
Febre & 5 & 1,8 \\
Outros & 49 & 18,2 \\
\hline
\end{tabular}

Ao longo do estudo, foram observadas 85 complicações, todas relacionadas à PVP, sendo as principais: dor local $(30,5 \% ; n=26)$, extravasamento $(25,9 \% ; n=22)$, cateter dobrado $(24,7 \%$; $n=21)$ e cateter tracionado $(18,8 \% ; n=16)$. Não foram identificadas complicações relacionadas à hipodermóclise.

\section{Discussão}

Outros estudos que avaliaram CVPs e as complicações associadas apresentaram participantes com características similares às do presente estudo, especialmente às relacionadas ao sexo, à idade média superior a 50 anos, ao ensino fundamental incompleto e à hipertensão arterial como a principal comorbidade..$^{3,11-13}$ 
Em relação às doenças de base apresentadas pelos participantes da pesquisa, pode-se constatar a similaridade com os dados do Instituto Nacional de Câncer (INCA), em que os tipos de câncer mais incidentes, em ambos os sexos, estimados para 2020 (exceto pele não melanoma), foram cólon e reto $(9,1 \%$ em homens, e 9,2\% em mulheres), seguido de traqueia, brônquio e pulmão $(7,9 \%$ em homens e 5,6\% em mulheres $) .{ }^{14}$

O tipo de câncer mais incidente em homens é o de próstata $(29,2 \%)$, e nas mulheres, o de mama (29,7\%). ${ }^{14}$ Em estudo prospectivo realizado com 10 pacientes sob CP com diagnóstico de câncer, sete pacientes foram diagnosticados com câncer de pulmão, um paciente com câncer de mama, um com câncer de próstata e um com câncer gastrointestinal, apresentando similaridade entre as doenças oncológicas de base encontradas na presente pesquisa. ${ }^{8}$

Os dados coletados apresentaram uma alta prevalência de HAS entre os participantes (47,1\%). As doenças crônicas não transmissíveis (DCNT) correspondem a $63 \%$ das causas de morte no mundo, equivalendo à principal causa de morbimortalidade. Cerca de um terço das mortes globais são causadas por doenças cardiovasculares, sendo a hipertensão arterial a mais prevalente. ${ }^{15}$ No Brasil, HAS autorreferida predomina na Região Sudeste, com 23,3\%. ${ }^{16}$ Esses dados justificam a HAS como principal comorbidade entre os participantes da pesquisa.

A Diabetes Mellitus foi a segunda comorbidade mais presente nos pacientes do presente estudo. Cerca de $80 \%$ da população mundial com Diabetes Mellitus vive em países de baixa e média renda. Segundo pesquisa descritiva que utilizou dados dos exames laboratoriais da Pesquisa Nacional de Saúde (PNS) entre 2014 e 2015, a prevalência de Diabetes Mellitus é mais elevada entre mulheres e aumenta conforme a idade. ${ }^{17}$

No presente artigo, a realização da PVP foi superior à realização da punção subcutânea. As características clínicas de pacientes sob CP e pacientes idosos, os quais apresentam o processo natural de envelhecimento das veias e perda de elasticidade da pele, dificultando a obtenção de acesso venoso, principalmente em pacientes com mais de 80 anos ou naqueles que já foram 
submetidos à quimioterapia, apontam para a indicação da hipodermóclise. ${ }^{6}$ Entretanto, apesar disso, a adesão à realização da hipodermóclise (punção subcutânea) foi baixa neste estudo.

As PVPs obtidas com apenas uma tentativa foram de 55,7\%, e 44,3\% foram obtidas com duas ou mais tentativas. Segundo a literatura, as chances de sucesso na primeira tentativa estão entre $10 \%$ e $55 \%$, sendo considerada uma técnica desafiante para os profissionais que a realizam. ${ }^{18}$ Dessa forma, o resultado da presente pesquisa encontra dados similares ao da literatura.

Revisão integrativa que identificou os fatores que dificultam a PVP em adultos concluiu que a dificuldade em obtê-la está associada a aspectos demográficos, como sexo e idade; dados clínicos, como comorbidades, estado nutricional, visibilidade e palpabilidade da rede venosa; questões relacionadas ao dispositivo, como calibre e modelo do cateter; e elementos relacionados à habilidade do profissional. ${ }^{19}$ No presente estudo, por conta das condições clínicas dos participantes, a habilidade dos profissionais que realizaram o procedimento foi o diferencial para o sucesso das punções obtidas logo na primeira tentativa, conferindo ao paciente menor desconforto relacionado à inúmeras picadas.

O principal local de escolha para inserção do CVP foi a veia metacarpiana dorsal $(20,8 \%)$, o que está em acordo com outras averiguações sobre PVP, que apontam o dorso da mão como o local mais utilizado para inserção de cateteres venosos. ${ }^{12-13}$ Em relação ao local da punção subcutânea, 60,0\% foram prioritariamente realizadas na região anterolateral da coxa, seguido da região abdominal $(25,0 \%)$ e região deltoidea $(15,0 \%)$. Em pesquisa realizada em Curitiba, Paraná, com pacientes em acompanhamento pela equipe de CP em atendimento ambulatorial e/ou domiciliar, de um total de 30 hipodermóclise, 28 foram realizadas na região infraclavicular, e duas, na região abdominal, regiões essas que diferiram das regiões escolhidas pelos profissionais no presente artigo, também consideradas adequadas e seguras., ${ }^{3,9}$

O cateter mais utilizado foi o não agulhado de teflon do tipo Abocath ${ }^{\circledast}$ (93,7\%). Pesquisa realizada comparando tipos de cateteres endovenosos concluiu que as taxas de risco acumulado 
para desenvolvimento de flebite, infiltração e tração são menores com o uso do cateter não agulhado de teflon do tipo Intima ${ }^{\oplus}{ }^{20}$ Entretanto, o cateter não agulhado do tipo Intima ${ }^{\varpi}$ foi o menos utilizado, fato provavelmente relacionado ao custo do cateter e por se tratar de serviço de saúde público e com recursos escassos.

Entre os cateteres, o calibre mais utilizado foi o de $22 \mathrm{G}(69,5 \%)$, seguido de $20 \mathrm{G}(23,0 \%)$ e $24 \mathrm{G}(7,5 \%)$. Em um serviço de cirurgia, onde foram analisados 58 pacientes com punções venosas periféricas internados em uma unidade hospitalar da Região Norte de Portugal, o calibre mais utilizado foi $18 \mathrm{G}$ (70,5\%), seguido de $20 \mathrm{G}$ e $24 \mathrm{G} .{ }^{11}$ Outro estudo identificou a prevalência no uso dos calibres de $22 \mathrm{G}(60,0 \%)$ e $20 \mathrm{G}(37,7 \%)$, seguido de $18 \mathrm{G}(2,3 \%)$ e $24 \mathrm{G}(0,4 \%) .{ }^{12}$ Em outras duas pesquisas de mesma natureza, houve prevalência do uso de cateteres de 20G. ${ }^{3,11}$

Ensaio clínico randomizado realizado com 169 participantes aponta que o uso do calibre 20G reduz o risco de ocorrência de complicações, quando comparado ao calibre $22 \mathrm{G}$, devido à menor incidência de infiltração. ${ }^{20}$ Entretanto, devem ser selecionados cateteres de menor calibre, pois reduzem a ocorrência de flebite por irritação da parede da veia pela cânula e reduzem a obstrução do fluxo sanguíneo dentro do vaso. ${ }^{21}$

Assim, 73,1\% das punções endovenosas apresentavam fita de estabilização do cateter. A estabilização do cateter preserva a integridade do acesso e previne o deslocamento e perda deste. ${ }^{21} \mathrm{~A}$ estabilização inadequada pode levar à má aderência da PVP à pele, o que permite o deslocamento parcial ou completo, devido à movimentação do cateter para dentro e para fora da veia. ${ }^{22}$ É possível que tal fato tenha propiciado a ocorrência das complicações, como tração do cateter, na presente pesquisa.

O tipo de cobertura predominante foi o filme transparente estéril $(91,3 \%)$. Toda a cobertura utilizada para cateter periférico deve ser estéril e transparente ou semioclusiva, a fim de proteger o sítio da punção, minimizar a possibilidade de infecção e fixar o dispositivo para prevenir movimentação e dano ao vaso. ${ }^{22}$ Em hospital de Portugal, foram analisados 110 pacientes com CVP acerca da presença de flebite ou infiltração, o tipo de cobertura mais utilizada foi também a película 
transparente estéril $(88,8 \%) .{ }^{12}$ Outro estudo realizado em Portugal identificou a baixa adesão ao uso do curativo estéril, impermeável e transparente entre a equipe de enfermagem. Tal fato contraria as medidas para prevenção de flebite e infecção, dificultando a avaliação do local de inserção do cateter, sendo necessária a troca do sistema de fixação diariamente para permitir a visualização e avaliação do sítio de inserção, o que, em diversas situações, resultava na exteriorização acidental do cateter e na necessidade de nova punção venosa. ${ }^{23}$

Apenas 12,9\% das punções, venosas ou subcutâneas estavam completas, $84,7 \%$ das identificações estavam incompletas e 2,5\% não possuíam identificação. Considera-se identificação adequada aquela que contempla informações, como nome do profissional que realizou a punção, data e hora da punção. ${ }^{24}$ Nessa definição, não foi incluído o tipo e calibre do cateter, uma vez que o filme transparente permite essa identificação visual. A prática de não identificação pode expor o paciente a risco de infecção e reações adversas, devido à instalação e manutenção inadequada, que podem resultar em aumento de permanência e de custo da internação hospitalar. ${ }^{24}$

Nesta pesquisa, as principais finalidades das punções foram a antibioticoterapia, presente em 33 casos (11,8\%), seguido de analgesia (11,4\%) e hidratação $(4,3 \%)$. Apesar desses resultados, a classe de medicamentos mais prescrita no presente estudo foi a dos analgésicos, sendo a dipirona o medicamento mais prescrito (12,1\%), seguido de soroterapia para hidratação $(10,7 \%)$. Pesquisa bibliográfica elencou as principais medicações utilizadas em $\mathrm{CP}$, evidenciando que o principal sintoma tratado é a dor, sendo os opioides e analgésicos as principais classes de medicamentos, ${ }^{25}$ resultado semelhante ao do presente estudo, em que a principal classe farmacológica prescrita foi a dos analgésicos.

Os principais motivos de internação foram dispneia, seguido de dor, adinamia, piora do estado geral e náuseas e vômito. Na Região Sul do Brasil, 213 pacientes com câncer, internados entre 2005 e 2008, foram abordados, a fim de descrever a estrutura e o processo de trabalho do Programa de Internação Domiciliar Interdisciplinar. Investigou-se o motivo de internação desses pacientes, 
identificando, entre os sintomas mais frequentes, anorexia, dor e astenia, além de desidratação, náuseas e vômitos e dispneia, motivos também presentes nos resultados da presente pesquisa. ${ }^{26}$

A dispneia é um dos sintomas mais comuns no contexto de oncológicos em CP, acometendo de 21 a 90\% dos pacientes, havendo ou não envolvimento pulmonar, sendo importante realizar a avaliação minuciosa da situação. ${ }^{27} \mathrm{~A}$ dor foi o segundo sintoma mais frequente nos participantes da pesquisa, apresentando uma alta prevalência. Em estudo qualitativo realizado com 14 cuidadores de pessoas com câncer avançado, relatou-se que $71 \%$ dos enfermos tiveram dor. ${ }^{28}$ Conforme o conceito de dor total, ela não é composta apenas pelo componente físico, incluindo também a dor psíquica, social e espiritual. Sendo assim, o tratamento adequado da dor é complexo e individual, por se tratar de algo subjetivo. ${ }^{29} \mathrm{Na}$ presente pesquisa, foram identificadas 85 complicações, todas relacionadas à PVP. As principais complicações apresentadas entre os pacientes participantes foram dor local (30,5\%), extravasamento $(25,9 \%)$, cateter dobrado $(24,7 \%)$ e cateter tracionado $(18,8 \%)$.

Hospital universitário de grande porte de Curitiba, Paraná, analisou a distribuição de complicações dos CVPs, encontrando a flebite (18,34\%) como principal complicação, seguida de infiltração (11,83\%), obstrução $(11,24 \%)$ e tração $(9,47 \%) .{ }^{20}$ Análise realizada com uma amostra de 92 cateteres trouxe flebite $(36,54 \%)$, infiltração $(23,08 \%)$, tração acidental do cateter $(17,31 \%)$, obstrução $(15,38 \%)$, infecção local $(3,85 \%)$, extravasamento $(1,92 \%)$ e tromboflebite $(1,92 \%)$ como principais complicações relacionadas ao $\mathrm{CVP} .{ }^{3}$ Dessa forma, todas as complicações relacionadas ao CVP encontradas no presente estudo estão presentes em outras pesquisas de natureza semelhante.

O presente estudo constatou a ausência de complicações relacionadas à hipodermóclise, porém encontra-se na literatura análises que descrevem tais ocorrências. Revisão integrativa identificou 13 artigos que abordavam os efeitos adversos da hipodermóclise em adultos. Os efeitos adversos mais relatados nos estudos analisados foram considerados de risco mínimo, reversíveis e de pouca importância clínica, como dor e edema no local da punção, celulite e 
absorção insuficiente da solução com acúmulo de líquido no local. Os efeitos adversos citados podem ser tratados por meio de massagem local, redução e mudança do sítio de punção. Tais ocorrências são relatadas, frequentemente, após três dias de tratamento em um único sítio subcutâneo, podendo também ter como causa a punção errônea. Por isso, recomenda-se que a técnica seja realizada por profissionais capacitados, sendo o sítio de inserção inspecionado regularmente e com o tempo de permanência entre 48 e 96 horas. Dessa forma, segundo a revisão, os efeitos adversos causados pela hipodermóclise em pacientes adultos são, na maioria das vezes, mínimos e reversíveis e não causam danos graves. ${ }^{6}$

Uma limitação observada foi o baixo número de hipodermóclises incluídas neste estudo, devido ao tempo reduzido para a realização da coleta de dados. Os achados permitem identificar a necessidade de disseminar o conhecimento dos benefícios da hipodermóclise e seus impactos na qualidade de vida de pacientes oncológicos sob CP, que passam por diversas intervenções dolorosas para que haja maior adesão ao procedimento pelos profissionais de saúde que os assistem.

\section{Conclusão}

A taxa de complicações relacionadas às PVPs foi alta, não sendo observadas em punções subcutâneas. Houve o uso predominante da PVP em detrimento do uso da via subcutânea, mesmo essa sendo uma alternativa viável e recomendada para os pacientes oncológicos sob CP.

O presente estudo não obteve uma quantidade significativa de hipodermóclises, reafirmando-a como uma técnica ainda pouco utilizada pelos profissionais da área da saúde, porém, quando realizadas, não apresentaram efeitos adversos, sendo caracterizada como uma técnica segura e sem complicações graves. A principal complicação encontrada no presente estudo foi dor local, facilmente resolvida por meio da mudança do local de sítio da punção. Assim, de forma geral, o risco de complicações apresentado foi mínimo e de pouca importância clínica, podendo ser facilmente tratado e solucionado. 


\section{Referências}

1. International Association for Hospice \& Palliative Care (IAHPC). Palliative care definition [Internet]. Houston (TX): International Association for Hospice \& Palliative Care; 2018 [cited 2020 Oct 28]. Available from: https://hospicecare.com/what-we-do/projects/consensus-based-definition-of-palliative-care/definition

2. Scales K. Use of hypodermoclysis to manage dehydration. Nurs Older People. 2011 Jun;23(5):16-22. doi: 10.7748/nop2011.06.23.5.16.c8528

3. Danski MTR, Oliveira GLR, Johann DA, Pedrolo E, Vayego SA. Incidência de complicações locais no cateterismo venoso periférico e fatores de risco associados. Acta Paul Enferm. 2015;28(6):517-23. doi: 10.1590/19820194201500087

4. Braga LM, Salgueiro-Oliveira AS, Henriques MAP, Arreguy-Sena C, Albergaria VMP, Parreira PMSD. Peripheral venipuncture: comprehension and evaluation of nursing practices. Texto Contexto Enferm. 2019;28:e20180018. doi: 10.1590/1980-265X-TCE-2018-0018

5. Bruno VG. Hipodermóclise: revisão de literatura para auxiliar a prática clínica. Einstein (São Paulo). 2015;13(1):122-8. doi: 10.1590/S1679-45082015RW2572

6. Nunes PMSA, Souza RCS. Adverse effects of hypodermoclysis in adult patients: an integrative review. REME Rev Min Enferm. 2016;20:e951. doi: 10.5935/1415-2762.20160020

7. Sociedade Brasileira de Geriatria e Gerontologia (SBGG). O uso da via subcutânea em geriatria e cuidados paliativos: um guia da SBGG e da ANCP para profissionais [Internet]. $2^{\mathbf{a}}$ ed. Rio de Janeiro: Sociedade Brasileira de Geriatria e Gerontologia; 2016 [acesso em 2020 set 30]. 56 p. Disponível em: https://sbgg.org.br/wp-content/uploads/2016/06/uso-da-via-subcutanea-geriatria-cuidados-paliativos.pdf

8. Perera AH, Smith $\mathrm{CH}$, Perera AH. Hipodermoclisis en pacientes con cáncer terminal. Rev Cuba Med [Internet]. 2011 [acceso 2020 out 01];50(2):150-6. Disponible en: http://scielo.sld.cu/scielo.php?script=sci_arttext\&pid=S0034-75232011000200005\&lng=es

9. Justino ET, Tuoto FS, Kalinke LP, Mantovani MF. Hipodermóclise em pacientes oncológicos sob cuidados paliativos. Cogitare Enferm. 2013;18(1):84-9. doi: 10.5380/ce.v18i1.31307

10. BRASIL. Ministério da Saúde. Conselho Nacional de Saúde. Resolução no 466, de 12 de dezembro de 2012. Dispõe sobre diretrizes e normas regulamentadoras de pesquisas envolvendo seres humanos. Diário Oficial da União, Brasília, DF, 13 jun. 2013. Seção 1, p. 59. Disponível em: http://bvsms.saude.gov.br/bvs/saudelegis/cns/2013/res0466_12_12_2012.html. Acesso em: 18 jun. 2017.

11. Danski MTR, Johann DA, Vayego SA, Oliveira GRL, Lind J. Complicações relacionadas ao uso do cateter venoso periférico: ensaio clínico randomizado. Acta Paul Enferm. 2016;29(1):84-92. doi:10.1590/1982-0194201600012

12. Braga LM, Parreira PM, Oliveira ASS, Mónico LSM, Arreguy-Sena C, Henriques MA. Phlebitis and infiltration: vascular trauma associated with the peripheral venous catheter. Rev Latinoam Enferm. 2018;26:e3002. doi: 10.1590/1518-8345.2377.3002 
13. Nobre ASP, Martins MDS. Prevalência de flebite da venopunção periférica: fatores associados. Referência; 2018; serIV(16):127-38. doi: 10.12707/RIV17058

14. Ministério da Saúde, Instituto Nacional de Câncer José Alencar Gomes da Silva (INCA). Estimativa 2020: incidência de câncer no Brasil [Internet]. Rio de Janeiro: INCA; 2019 [acesso em 2020 set 30]. Disponível em: https://www.inca.gov.br/sites/ufu.sti.inca.local/files/media/document/estimativa-2020incidencia-de-cancer-no-brasil.pdf

15. Malta DC, Bernal RTI, Andrade SSCA, Silva MMA, Velasquez-Melendez G. Prevalence of and factors associated with self-reported high blood pressure in Brazilian adults. Rev Saúde Pública. 2017;51(Suppl 1):11s. doi: 10.1590/S1518-8787.2017051000006

16. Malachias MVB, Souza WKSB, Plavink FL, Rodrigues CIS, Brandão AA, Neves MFT, et al. 7th Brazilian guideline of arterial hypertension: chapter 1 - concept, epidemiology and primary prevention. Arq Bras Cardiol. 2016;107(3 Suppl 3):1-6. doi: 10.5935/abc.20160151

17. Malta DC, Duncan BB, Schmidt MI, Machado IE, Silva AG, Bernal RTI, et al. Prevalence of diabetes mellitus as determined by glycated hemoglobin in the Brazilian adult population, National Health Survey. Rev Bras Epidemiol. 2019;22(Suppl 2):E190006. doi: 10.1590/1980-549720190006.supl.2. eCollection 2019

18. Oliveira AM, Danski MTR, Pedrolo E. Punção venosa periférica guiada por ultrassonografia: prevalência de sucesso e fatores associados. Cogitare Enferm. 2017;22(3):e49599. doi: 10.5380/ce.v22i3.49599

19. Marinho AM, Sabino FHO, Monteiro DAT, Figueira VSA, Azevedo GN, Toffano SEM. Difficult peripheral venous puncture in adults: integrative review. Rev Enferm UERJ. 2019;27:e42567. doi: https://doi.org/10.12957/reuerj.2019.42567

20. Johann DA, Danski MTR, Vayego SA, Barbosa DA, Lind J. Risk factors for complications in peripheral intravenous catheters in adults: secondary analysis of a randomized controlled trial. Rev Latinoam Enferm. 2016;24:e2833. doi: 10.1590/1518-8345.1457.2833

21. Gerência de Vigilância e Monitoramento em Serviços de Saúde (GVIMS), Gerência Geral de Tecnologia em Serviços de Saúde (GGTES). Medidas de prevenção de infecção relacionada à assistência à saúde [Internet]. Brasília (DF): Agência Nacional de Vigilância Sanitária; 2017 [acesso em 2020 out 06]. Disponível em: http://www.riocomsaude.rj.gov.br/Publico/MostrarArquivo.aspx?C=pCiWUy84\%2BR0\%3D

22. Marsh N, Webster J, Mihala G, Rickard CM. Devices and dressings to secure peripheral venous catheters to prevent complications. Cochrane Database Syst Rev. 2015 jun 12;6:CD011070. doi: 10.1002/14651858.CD011070.pub2

23. Salgueiro-Oliveira AS, Basto ML, Braga LM, Arreguy-Sena C, Melo MN, Parreira PMSD. Nursing practices in peripheral venous catheter: phlebitis and patient safety. Texto Contexto Enferm. 2019;28:e20180109. doi: 10.1590/1980-265x-tce-2018-0109

24. Souza VS, Amorim DO, Silva NB, Stevanato KP, Melo WA, Costa MAR. Indicadores de qualidade da assistência de enfermagem na terapia intravenosa periférica. Rev Enferm UFPE On Line. 2017 Mai;11(Suppl 5):1989-95. doi: 10.5205/1981-8963-v11i5a23352p1989-1995-2017 
25. Guerra ED, Souza Júnior JGC, Silva JPS, Lima CT, Andrade LVB, Silva AP, et al. Principais medicações utilizadas em cuidados paliativos: revisão de literatura. Braz J Dev. 2019;11(5):26862-76. doi: 10.34117/bjdv5n11-308

26. Fripp JC, Facchini LA, Silva SM. Caracterização de um programa de internação domiciliar e cuidados paliativos no Município de Pelotas, Estado do Rio Grande do Sul, Brasil: uma contribuição à atenção integral aos usuários com câncer no Sistema Único de Saúde, SUS. Epidemiol Serv Saúde. 2012;21(1):69-78. doi: 10.5123/S167949742012000100007

27. Carvalho RT. Dispneia, tosse e hipersecreção de víaas aéreas. In: Academia Nacional de Cuidados Paliativos (ANCP). Manual de Cuidados Paliativos [Internet]. 2a ed. São Paulo: Academia Nacional de Cuidados Paliativos (ANCP); 2012 [acesso em 2020 out 30]. p. 151-67. Disponível em: http://biblioteca.cofen.gov.br/wp-content/uploads/2017/05/Manual-de-cuidados-paliativos-ANCP.pdf

28. Portela FR, Modena CM. Pacientes com câncer avançado: o acesso aos opioides e demais medicamentos para controle da dor. Rev Bras Cancerol. 2018;64(2):195-01. doi: 10.32635/2176-9745.RBC.2018v64n2.78.

29. Gomes ALZ, Othero MB. Cuidados paliativos. Estud Av. 2016;30(88):155-66. doi: 10.1590/S010340142016.30880011

\section{Editora Científica: Tânia Solange Bosi de Souza Magnago \\ Editora Associada: Silvana Bastos Cogo}

Fomento / Agradecimento: Apoio do Programa Unificado de Bolsas da Universidade de São Paulo através de Auxílio a Projeto de Pesquisa.

\section{Autor correspondente}

Fabiana Bolela de Souza

E-mail: fbolela@usp.br

Endereço: Rua Legionario Maurício 234, Ipiranga, Ribeirão Preto, SP. CEP: 14060-310

\section{Contribuições de Autoria}

1 - Ana Julia de Oliveira Lago

Análise e/ou interpretação dos dados, revisão final com participação crítica e intelectual no manuscrito.

\section{2 - Ana Carolina de Souza}

Revisão final com participação crítica e intelectual no manuscrito.

\section{3 - Fabiana Bolela de Souza}

Concepção ou desenho do estudo/pesquisa, análise e/ou interpretação dos dados, revisão final com participação crítica e intelectual no manuscrito.

\section{Como citar este artigo}

Lago AJO, Souza ACS, Souza FB. Complications related to peripheral venipuncture and hypodermoclysis in cancer patients under palliative care. Rev. Enferm. UFSM. 2021 [Access on: Year Month Day]; vol.11 e76: 1-18. DOI: https://doi.org/10.5902/2179769264392 